\title{
Minimal incomplete norms on Banach algebras
}

by

\author{
MICIAEL, J. MEYER (Atlanta, Ga.)
}

Abstract. Wo study the family of all not necessarily complete algebra norms on a semisimple Banach algebra as a partially ordered set and investigate the existence and properties of minimal elements.

Introduction. Let $\mathcal{A}$ be a semisimple algebra over the field of complex numbers. Recall that an algebra norm on $\mathcal{A}$ is a norm which also satisfies $\|x y\| \leq\|x\|\|y\|$ for all $x, y \in \mathcal{A}$. From now on all norms will be assumed to be algebra norms. A famous theorem of $\mathrm{B}$. Johnson states that any two complete norms on $\mathcal{A}$ are equivalent. We will investigate what can be said if completeness of one or both the norms is dropped or replaced by some other condition. (The conclusion of equivalence is then weakened considerably.) A similar investigation is conducted in [12]. Our methods differ from [12] in that we study the family of norms on $\mathcal{A}$ as a partially ordered set and investigate minimal elements.

Let | |, || || be norms on $\mathcal{A}$. Following [12] let us say that || || majorizes | | if

and say that \|\| dominates $\mid$ if

$$
|x| \leq\|x\| \quad \text { for all } x \in \mathcal{A} \text {, }
$$

$$
|x| \leq C\|x\| \quad \text { for all } x \in \mathcal{A} \text { and some constant } C<\infty \text {. }
$$

Majorization defines a partial order on the family of all norms on $\mathcal{A}$. Domination defines a partial order on the family of classes of equivalent norms, that is, on the farnily of norm topologies on $\mathcal{A}$. Even though $(1)$ is more interesting we have to work with (0) which is more amenable to minimality arguments. A connection is given as follows:

Proposition 1. Let ||, || || be norms on $\mathcal{A}$ and suppose \|\| dominates ||. Then there is a norm $\mid$ lo on $\mathcal{A}$ which is equivalent to $\|$ and such that || || majorizes | |o.

1991 Mathematics Subject Classification: Primary 46H05.

Key words and phrases: Banech algebras, norms; spectral radius. 
This follows if [3, Theorem 1.4.1] is applied to the bounded semigroup $B_{1}(\|\|)=\{x \in \mathcal{A}:\|x\| \leq 1\}$ in the normed algebra $(\mathcal{A},||)$. See also [11]. From now on we consider the partial order $(0)$. A norm \|\| is minimal in this order if it satisfies

(2) $|x| \leq\|x\|$ for all $x \in \mathcal{A}$ implies $|x|=\|x\|$ for all $x \in \mathcal{A}$,

for all norms || on $\mathcal{A}$. Minimality of norms in the sense of majorization has been considered by several authors. In [2] it is shown that the complete - norm on $\mathcal{A}$ is minimal whenever $\mathcal{A}$ is a $C^{*}$-algebra, a closed subalgebra of the algebra $\mathcal{B}(X)$ of all bounded linear operators on a Banach space $X$ which contains all finite rank operators, or a modular annihilator $B^{\text {HW }}$-algebra, and in [5] an explicit description of all minimal norms on certain subalgebras of $\mathcal{B}(X)$ is derived. Minimality of the complete norm on a $C^{*}$-algebra is also used in [11].

Call two norms \|\|$,||$ on $\mathcal{A}$ consistent if the identity map $(\mathcal{A},\|\|) \rightarrow$ $(\mathcal{A}, \|)$ has closed graph. Equivalently \|\|$, \|$ are consistent if the set

$$
\mathcal{C}=\left\{a \in \mathcal{A}:\left\|x_{n}\right\| \rightarrow 0 \text { and }\left|a-x_{n}\right| \rightarrow 0\right.
$$

$$
\text { for some sequence } \left.\left(x_{n}\right) \subseteq \mathcal{A}\right\}=(0) \text {. }
$$

Notice that $\mathcal{C}$ is a two-sided ideal in $\mathcal{A}$ which is closed in both norms $\|$, | | [10, II.5]. $\mathcal{C}$ is called the consistency ideal of \|\|$, \mid$ |.

Two noncomplete norms on $\mathcal{A}$ camnot in general be expected to be equivalent. However, they may satisfy one of the following weakenings of equivalence:

(A) \|\|$,||$ are consistent.

(B) The closed unit ball of \|\| is closed in $\mid 1$.

(C) \|\|$_{2}||$ dominate a common norm.

(D) || dominates || ||

It is clear that $(D) \Rightarrow(B) \Rightarrow(A)$ and $(D) \Rightarrow(C) \Rightarrow(A)$. It is shown in [12, Lemma 3.2] that $(A) \Rightarrow(C)$ if one of the norms is complete. Below we give conditions on the norms \|\|$, \|$ as well as the algebra $\mathcal{A}$ which imply one of the conclusions (A) - (D).

Call a norm \|\| on $\mathcal{A}$ a $Q$-norm [12] if it satisfies $\|x\| \geq \varrho_{\mathcal{A}}(x)$ for all $x \in \mathcal{A}$, where $\varrho_{\mathcal{A}}$ denotes the spectral radius in $\mathcal{A}$. This property is often a sufficient substitute for completeness [8]. Let us call a normed algebra $\mathcal{A}$ a $Q$-normed algebra if the norm on $\mathcal{A}$ is a $\mathrm{Q}$-norm.

Let us first investigate minimal elements for the partial order $(0)$ on the family of Q-norms only: A Q-norm || || is minimal in this order iff it satisfies (2) for all $Q$-norms || on $\mathcal{A}$.

THEOREM 1. Suppose that $\mathcal{A}$ is a semisimple Banach algebra. Then (A) Any two $Q$-norms on $\mathcal{A}$ are consistent.
(B) Every Q-norm \|| $\|$ on $\mathcal{A}$ majorizes a minimal Q-norm

(C) The closed unit ball of a minimal Q-norm is closed in any other Q-norm on $\mathcal{A}$.

(D) A minimal Q-norm is continuous with respect to any other Q-norm in which $\mathcal{A}$ is a barreled space and in particular therefore with respect to the complete norm on $\mathcal{A}$.

(E) The completion of $\mathcal{A}$ in a minimal Q-norm is semisimple.

(F) Let $\|$ \|t be a minimal Q-norm on $\mathcal{A}$ and denote, for each primitive ideal $P$ in $\mathcal{A}$, by \|\|$_{r}$, the quotient norm on $\mathcal{A} / P$. Then $\|x\|=\sup _{P}\|x\|_{P}$ for all $x \in \mathcal{A}$.

Remark. (A) is well known. T. W. Palmer (personal communication) noticed that an argument of $T$. J. Ransford [9] can be modified so as to yield a simple proof of (A). We include the argument for the convenience of the reader. Notice that (C) represents a considerable strengthening of (A) for minimal Q-norms. (D) and (E) show that a minimal $Q$-norm is smooth in the sense of [12]. Thus Theorem 1 implies [12, Theorem 3.4]. All the algebras loc. cit. are permanent Q-algebras, that is, all norms are Q-norms.

Call a Banach algebra $\mathcal{A}$ with complete norm | | a weak $B^{\#-a l g e b r a ~ i f ~}$ for each $a \in \mathcal{A}$ with $|a|=1$ there exists $b \in \mathcal{A}$ with $|b|=1$ and $\varrho_{\mathcal{A}}(a b) \geq$ $\varepsilon$, where $\varepsilon>0$ is a constant independent of $a, b$. Notice that $\mathcal{A}$ is then semisimple.

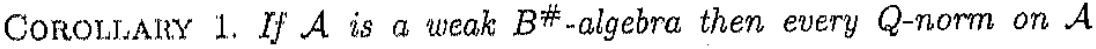
dominates the complete norm.

The weak $B^{H^{*}}$ condition is satisfied for all closed subalgebras $\mathcal{A} \subseteq \mathcal{B}(X)$ which act uniformly transitively on $X$ in the following sense: for each $x, y \in$ $X$ with $\|x\|=\|y\|=1$ there exists $A \in \mathcal{A}$ with $|A|_{\text {op }} \leq C$ and $A x=y$, where $C$ is a constant independent of $x, y$. This is always true if $\mathcal{A}$ contains the finite rank operators.

Corollary 1 should now be compared with the following result by $\mathrm{B}$. Yood [13]: If $\mathcal{A}$ is a closed subalgebra of $\mathcal{B}(X)$ which contains the finite rank operators, then every norm on $\mathcal{A}$ dominates the operator norm. Our conclusion is weaker but we do not assume that $\mathcal{A}$ has a socle.

We now wish to consider the family of all (submultiplicative) norms on A. The pointwise infimum of a chain of norms is a seminorm but it may have a nontrivial kernel. Thus in order to ensure the existence of minimal norms we have to impose some condition on the underlying algebra.

Define for $a \in \mathcal{A}$ the permanent radius $\varrho_{\mathrm{p}}(a)$ by $\varrho_{\mathrm{p}}(a)=\inf _{\phi} \varrho_{B}(\phi(a))$, where the infimum is taken over all (not necessarily continuous) embeddings $\phi$ of $\mathcal{A}$ into another Banach algebra $\mathcal{B}$. Thus

$$
\varrho_{\mathrm{p}}(a)=\inf \lim _{n \uparrow \infty}\left\|a^{n}\right\|^{1 / n},
$$


where the infimum is taken over all norms on $\mathcal{A}$. [3, I.4.2, p. 18] shows that in fact $\varrho_{\mathrm{p}}(a)=\inf \|a\|$, where the infimum is taken over all norms on $\mathcal{A}$.

Now let us say that the Banach algebra $\mathcal{A}$ satisfies condition $(P)$ if every nonzero closed ideal $l$ in $\mathcal{A}$ contains an element $a$ with $\varrho_{\mathrm{P}}(a)>0$. Examples of elements of positive permanent radius are furnished by the following

Proposition 2 ([7]). Let $\mathcal{A}$ be a Banach algebra.

(1) If $a \in \mathcal{A}$ is a quasidivisor of zero ( $a x=x$ or $x a=x$ for some nonzero $x \in \mathcal{A})$ then $\varrho_{\mathrm{p}}(a) \geq 1$.

(2) If $a, h \in \mathcal{A}$ are such that $a h=h a$ is a quasidivisor of zero then $\varrho_{\mathrm{p}}(a) \geq 1 / \varrho_{\mathcal{A}}(h)$

(3) $\varrho_{\mathrm{p}}(a) \geq \operatorname{dist}(C, 0)$, where $C$ is any connected component of $\operatorname{Sp}_{\mathcal{A}}(a)$.

Property $(P)$ guarantees the compactness of certain families of norms and thus the existence of minimal norms. We have the following facts:

THEOREM 2. Suppose that the Banach algebra $\mathcal{A}$ has property $(P)$.

(A) Any two norms on $\mathcal{A}$, one of which is a $Q$-norm, are consistent.

(B) For every (finitely valued) function $f: \mathcal{A} \rightarrow[0,+\infty)$, the family $\mathcal{N}_{f}$ of all norms on $\mathcal{A}$ which satisfy $\|x\| \leq f(x)$ for all $x \in \mathcal{A}$ is compact in the topology of pointwise convergence on $\mathcal{A}$.

(C) Every norm on $\mathcal{A}$ majorizes a minimal norm.

(D). The closed unit ball of a minimal norm is closed in any other norm with which the minimal norm is consistent.

(E) Every minimal norm is continuous with respect to the complete norm.

(F) If $\mathcal{B}$ is the completion of $\mathcal{A}$ in a minimal norm, then every nonzero closed ideal in $\mathcal{B}$ has nonzero intersection with $\mathcal{A}$.

$(G)$ The completion of $\mathcal{A}$ in a minimal norm also has property $(P)$.

Corollary 2. Suppose that $\mathcal{A}$ is minimally normed, that is, the complete norm on $\mathcal{A}$ is itself minimal. Then the following are equivalent:

(A) All norms on $\mathcal{A}$ are consistent with the complete norm.

(B) All norms on $\mathcal{A}$ are consistent.

(C) All norms on $\mathcal{A}$ are $Q$-norms.

(D) All norms on $\mathcal{A}$ dominate the complete norm.

(E) $\mathcal{A}$ has property $(P)$.

If $\mathcal{A}$ is a $C^{*}$-algebra or a closed subalgebra $\mathcal{A} \subseteq \mathcal{B}(X)$ which contains the finite rank operators, it is not hard to show that $\mathcal{A}$ is minimally normed [2] and has property (P) [6]. Thus Corollary 2 yields a new proof that all norms on $\mathcal{A}$ dominate the complete norm. This was shown previously in [4] for $C^{*}$-algebras and in [13] for closed subalgebras $\mathcal{A} \subseteq \mathcal{B}(X)$ which contain the finite rank operators.
2. Proofs. We need the following lemma by T. J. Ransford [9]. Completeness of the norm can be relaxed to the Q-property without change in the proof.

L.TMMA 1. S'uppose that $(\mathcal{A},\|\|)$ is a Q-normed algebra and that $p(\lambda):=$ $\sum a_{k} \lambda^{k}$ is a polynomial with coefficicints $a_{k} \in \mathcal{A}$. Then for every $R>0$ we have

$$
\mathscr{Q A}_{\mathcal{A}}(p(1))^{2} \leq \sup _{|\lambda|=2 R} Q_{\mathcal{A}}(p(\lambda)) \sup _{|\lambda| \mid=1 / R} Q_{\mathcal{A}}(p(\lambda)) .
$$

LeEMMA 2. Lat \|\| be a norm on $\mathcal{A}, \mathcal{B}$ the \|\| -completion of $\mathcal{A}$ and $I \subseteq \mathcal{B}$ an ideal in $\mathcal{B}$. Note that $\|x\|_{I}:=$ inf $f_{b \in l}\|x+b\|, x \in \mathcal{A}$, defines a seminorm on $\mathcal{A}$ with \|\|$_{1} \leq\|\|$. If \|\|$_{I}=\|\|$ then $I=(0)$.

Proof. Assume that $\|x\|_{L}=\|x\|$ for all $x \in \mathcal{A}$ and let $b \in I$. Since $\mathcal{A} \subseteq \mathcal{B}$ is dense we can choose a sequence $\left(x_{m}\right) \subseteq \mathcal{A}$ such that $x_{n} \rightarrow b$ in $\mathcal{B}$. Then for all $n \geq 1$ we have

$$
\left\|x_{n}\right\|=\left\|x_{n}\right\|_{I} \leq\left\|x_{n}-b\right\| \rightarrow 0
$$

i.e. $x_{n} \rightarrow 0$ in $\mathcal{A}$. It follows that $b=0$.

LEMMA 3 (Aupetit [1]). Let $\mathcal{A}, \mathcal{B}$ be Qnnormed algebras, $\phi: \mathcal{A} \rightarrow \mathcal{B}$ a homomorphism writh dense range and $a \in \mathcal{A}$. If there exists a sequence $\left(x_{n}\right) \subseteq \mathcal{A}$ such that $x_{n} \rightarrow 0$ in $\mathcal{A}$ and $\phi\left(x_{n}\right) \rightarrow \phi(a)$ in $\mathcal{B}$, then $\varrho_{\mathcal{B}}(\phi(a))=0$.

This is contained in [1]. T. J. Ransford gave a striking simplification of the proof in [9]. The argument is very similar to the proof of Theorem 1(A) below.

Proof of Theorem 1 . (A) Let $\mathcal{C}$ be the consistency ideal of the Q-norms \|\| and \|\|$_{1}$. We wish to show that $\mathcal{C}=(0)$. Let $a \in \mathcal{C}$ and choose $x_{n} \in \mathcal{A}$ such that $\left\|x_{n}\right\| \rightarrow 0$ and $\left\|x_{n}-a\right\|_{1} \rightarrow 0$. Set $p(\lambda):=\lambda x_{n}+a-x_{n}$. Then $p(1)=a$ and by Ransford's lemma for every $R>0$

$$
\begin{aligned}
& Q_{\mathcal{A}}(a)^{2} \leq \sup _{|\lambda|=a n} Q \mathcal{A}\left(\lambda x_{n}+a-x_{n}\right) \sup _{|\lambda|=\infty 1 / R} Q_{\mathcal{A}}\left(\lambda x_{n}+a-x_{n}\right) \\
& \leq \sup _{|\lambda| m_{n} R}\left\|\lambda x_{n}+a-x_{n}\right\| \sup _{|\lambda| x_{1} / R}\left\|\lambda x_{n}+a-x_{n}\right\|_{1} \\
& \leq\left[(R+1)\left\|x_{n}\right\|+\|a\|\right]\left[\frac{1}{R}\left\|x_{n}\right\|_{1}+\left\|a-x_{n}\right\|_{1}\right] .
\end{aligned}
$$

Let $n \uparrow \infty$ to obtain

$$
Q_{\mathcal{A}}(a)^{2} \leq\|a\| C / R
$$

where $C:=\sup _{n}\left\|x_{n}\right\|_{1}$. Now let $R \uparrow \infty$ to obtain $\varrho_{\mathcal{A}}(a)^{2}=0$. Thus $C$ is an ideal in $\mathcal{A}$ on which the spectral radins vanishes. Now semisimplicity of $\mathcal{A}$ implies $\mathcal{C}=(0)$. 
(B) The (pointwise) infimum of a chain $\mathcal{C}=\left\{\|\|_{\lambda}\right\}$ of $Q$-norms is easily seen to be a $Q$-norm. Thus (B) follows from Zorn's lemma.

(C) Let \|\| be a minimal $Q$-norm on $\mathcal{A}$ and $B=\{x \in \mathcal{A}:\|x\| \leq 1\}$ its closed unit ball. Suppose that \|\|$_{1}$ is another $Q$-norm, let $D$ be the \|\|$\|_{1}$-closure of $B$ and \|\|$\|$ the Minkowski functional of $D$,

$$
\|\mid a\|=\inf \left\{t>0: t^{-1} a \in D\right\}
$$

Since $D$ is an absolutely convex semigroup in $\mathcal{A}$ it follows that \|\|$\|$ is a submultiplicative seminorm on $\mathcal{A}$. Suppose that $a \in \mathcal{A}$ and $\|a\| \|=0$. Then $s a \in D$ for all $s>0$. For every integer $n>0$ choose $x_{n} \in B$ with $\left\|x_{n}-n a\right\|_{1}<1$. Then $\left\|x_{n} / n\right\| \rightarrow 0$ and $\left\|x_{n} / n-a\right\|_{1} \rightarrow 0$ and so $a$ is in the consistency ideal of \|\|$,\|\|_{1}$. It follows from (A) that $a=0$. Thus \|\|$\|$ is a norm on $\mathcal{A}$. We wish to show that it is a Q-norm. Since $D$ is its unit ball, it will suffice to show that $a \in D$ implies $\varrho_{\mathcal{A}}(a) \leq 1$.

Let $a \in D$ and choose $x_{n} \in B\left(\left\|x_{n}\right\| \leq 1\right)$ such that $\left\|a-x_{n}\right\|_{1} \rightarrow 0$. Multiplying the $x_{n}$ with suitable scalars $\alpha_{n}<1, \alpha_{n} \rightarrow 1$, we may assume that $\left\|x_{n}\right\|_{1} \leq\|a\|_{1}$ for all $n \geq 1$. Using Ransford's lemma and arguing as in the proof of (A) we obtain for every $R>0$ the inequality

$$
\varrho_{\mathcal{A}}(a)^{2} \leq\left[(R+1)\left\|x_{n_{2}}\right\|+\|a\|\right]\left[\frac{1}{R}\left\|x_{n_{2}}\right\|_{1}+\left\|a-x_{n_{1}}\right\|_{1}\right]
$$

Fix $R$ and let $n \uparrow \infty$ to obtain

$$
\varrho_{\mathcal{A}}(a)^{2} \leq[(R+1)+\|a\|] \frac{1}{R}\|a\|_{1} .
$$

Now let $R \uparrow \infty$ to obtain $\varrho_{\mathcal{A}}(a)^{2} \leq\|a\|_{1}$. Since $D$ is a semigroup we may replace $a$ by any power $a^{n}$ to obtain $\varrho_{\mathcal{A}}(a)^{2} \leq\left\|a^{n}\right\|_{1}^{1 / n}$. Let $n \uparrow \infty$. If $\mathcal{B}$ denotes the completion of $\mathcal{A}$ in \|\|$_{1}$ we obtain $\varrho_{\mathcal{A}}(a)^{2} \leq \varrho_{\mathcal{B}}(a) \leq \varrho_{\mathcal{A}}(a)$ and hence $\varrho_{\mathcal{A}}(a) \leq 1$.

Thus \|\|\|\| is a $Q$-norm on $\mathcal{A}$ and clearly \|\|$\|\leq\| \|$. By minimality of \|\| we have \|\|$\|=\| \|$ and hence $D=B$. Thus $B$ is \|\|$_{1}$-closed.

(D) Let \|\| be a minimal $Q$-norm and \|\|$_{1}$ any $Q$-norm on $\mathcal{A}$. By (C) the closed unit ball $B=\{a \in \mathcal{A}:\|a\| \leq 1\}$ of \|\| is closed and hence a barrel in the algebra $\left(\mathcal{A},\|\|_{1}\right)$. Tf this latter space is barreled, $B$ is a $\|$ || -neighborhood of zero. It follows that \|\| is continuous with respect to \|\|$_{1}$.

(E) Let \|\| be a minimal $Q$-norm on $\mathcal{A}, \mathcal{B}$ the \|\| -completion of $\mathcal{A}$ and $J=\operatorname{rad}(\mathcal{B})$ the Jacobson radical of $\mathcal{B}$. Since \|\| is a $Q$-norm, we have for all $x \in \mathcal{A}, b \in J, \varrho_{\mathcal{A}}(x)=\varrho_{\mathcal{B}}(x)=\varrho_{\mathcal{B}}(x+b) \leq\|x+b\|$. Thus, in the notation of Lemma 2,

$$
\|x\|_{J}=\inf _{b \in J}\|x+b\|_{,} \quad x \in \mathcal{A}
$$

is a $Q$-norm on $\mathcal{A}$. Clourly \|\|$_{J} \leq\|\|$. By minimality it follows that \|\|$_{y}=\|\|$. Leenuma 3 now implies that $J=(0)$.

(F) Let \|\| be a minimal $Q$-norm on $\mathcal{A}$. Every primitive ideal $P$ in $\mathcal{A}$ is \|\| -closed and the quotient norm \|\|$_{P}$, on $\mathcal{A} / P$ is again a $Q$-norm [8]. By semisimplicity of $\mathcal{A}_{3}||=\sup _{F},\|\|$ is a norm on $\mathcal{A}$. For each $x \in \mathcal{A}$ we have

$$
Q_{A}(x)=\operatorname{sip}_{P^{2}} Q \mathcal{A} / P(x+P) \leq \sup _{P}\|x+P\|_{p}=|x| .
$$

Thus || is a $Q$ nilorm on $\mathcal{A}$. Since ||$\leq\|\|$, we have ||$=\|\|$, by minimality of $\|$ H.

Proof of theorem 2. (A) Let \|\|$, \|$ be norms on $A$, assume that \|\| is a $Q$-norm and let $\mathcal{B}$ be the | |-completion of $\mathcal{A}$. By Theorem 1 there exists a continuous Qmorm (for example a minimal Q-norm) $\left\|_{0}\right\|_{0}$ such that \|\|$_{0} \leq\|\|$. The respective consistency ideals satisfy $\mathcal{C}(\|\|,||,) \subseteq \mathcal{C}\left(\|\|_{0}, \mid,\right)$. Thus consistency of \|\|$_{0,}, \mid$ implies consistency of \|\|$, \|$. We may therefore assume that $\|$ || is continuotis.

Let $\mathcal{C}$ be the consistency ideal of \|\|$, \mid$. Then $\mathcal{C}$ is closed in both norms and thus in the complete norm on $\mathcal{A}$. Let $a \in \mathcal{C}$. Then there exists a sequence $\left(x_{n}\right)$ in $\mathcal{A}$ stch that $x_{n} \rightarrow 0$ in the Q-normed algebra $(\mathcal{A},\|\|)$ and $x_{n} \ldots a$ in the Banach algebra $\mathcal{B}$. Lemma 3 applied to the inclusion homomorphism. $\mathcal{A} \rightarrow \mathcal{B}$ yields $Q_{p}(a) \leq Q B(a)=0$. Thus $\mathcal{C}$ does not contain elements $a$ with $Q_{p}(a)>0$. By assumption on the Banach algebra $\mathcal{A}$ we must have $C=(0)$.

(B) It will suffice to show that $\mathcal{N}_{f}$ is closed in the compact product space $Z=\prod_{x \in \mathcal{A}}[0, f(x)]$ (in which it is contained). Suppose that \|\| is in the clostre of $\mathcal{N}_{f}$ in $Z$ and choose a net $\left(\|\|_{\lambda}\right) \subseteq Z$ such that $\|x\|_{\lambda} \rightarrow\|x\|$ for all $x \in \mathcal{A}$. Then \|\| is a seminorm on $\mathcal{A}$ which satisfies $\|x\| \leq f(x)$ for all $x \in \mathcal{A}$. It remains to be shown only that $I=\operatorname{ker}(\|\|)=(0)$.

Assume $I \neq(0)$ and let $J=\tilde{I}$ be the closure of $I$ in the complete norm of $\mathcal{A}$. By assumption on $\mathcal{A}$, contains an element $a$ with $\varrho_{\mathrm{p}}(a)>0$. We wish to derive a contradiction.

Let $\mathcal{B}$ be the completion of $\mathcal{A}$ in the seminorm \|\| and $\phi: \mathcal{A} \rightarrow \mathcal{B}$ the natural homomorphism. $B$ is constructed as usual as the algebra of equivalence classes of \|\| -Cauchy sequences in $\mathcal{A}$ and this identifies elements in the kernel of \|\| . Thus $\mathcal{B}$ ts a Banach algebra and the homomorphism $\phi$ satisfles $\|\phi(x)\|=\|x\|$ and thus $\operatorname{ker}(\phi)=\operatorname{ker}(\|\|)$.

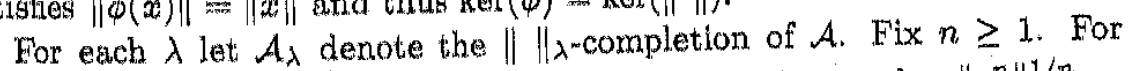
each $\lambda$ we have $\left\|a^{m_{n}}\right\|_{\lambda}^{1 / n} \geq Q_{A_{\lambda}}(a) \geq Q_{p}(a)$, and hence also $\left\|a^{n}\right\|^{1 / n}=$ $\lim _{\lambda}\left\|a^{n}\right\|_{\lambda}^{1 / n} \geq Q_{\mathrm{p}}(a)$. It follows that

$$
Q_{\mathcal{B}}(\phi(a))=\lim _{n+\uparrow \infty}\left\|\phi(a)^{n}\right\|^{1 / n}=\lim _{n \uparrow \infty}\left\|a^{n}\right\|^{1 / n} \geq \varrho_{\mathrm{p}}(a)>0 .
$$


On the other hand, choose a sequence $\left(a_{n}\right) \subseteq I=\operatorname{ker}(\phi)$ such that $a_{n} \rightarrow a$ in $\mathcal{A}$ and set $x_{n}:=a-a_{n}$. Then $\phi\left(x_{n}\right)=\phi(a)$ for all $n \geq 1$. We have $x_{n} \rightarrow 0$ in $\mathcal{A}$ and $\phi\left(x_{n}\right) \rightarrow \phi(a)$ in $\mathcal{B}$. Now Lemma 3 implies that $\varrho_{\mathcal{B}}(\phi(a))=0$. This is the desired contradiction.

(C) It will suffice to show that every chain $\left\{\|\|_{\lambda}\right\}$ of norms on $\mathcal{A}$ is bounded below by a norm on $\mathcal{A}$. Assume \|\|$_{\lambda} \leq\|\|_{\mu}$ whenever $\lambda \leq \mu$ and fix an index $\lambda_{0}$. For each $\lambda \leq \lambda_{0}$ let $\mathcal{N}_{\lambda}$ denote the family of all norms on $\mathcal{A}$ which satisfy $\|x\| \leq\|x\|_{\lambda}$ for all $x \in \mathcal{A}$. Then $\left\{\mathcal{N}_{\lambda}: \lambda \leq \lambda_{0}\right\}$ is a family of closed subsets of the compact set $\mathcal{N}_{\lambda_{0}}$ which has the finite intersection property. Thus $\bigcap_{\lambda \leq \lambda_{0}} \mathcal{N}_{\lambda} \neq \emptyset$. Every norm in this intersection is a lower' bound for the chain $\left\{\|\|_{\lambda}\right\}$.

(D), (E) As in Theorem 1.

(F) Suppose that $\mathcal{B}$ is the completion of $\mathcal{A}$ in a minimal norm \|\| and $I$ a closed ideal in $\mathcal{B}$. Then $\|a\|_{I}=\inf _{x \in I}\|a-x\|$ defines a seminorm on $\mathcal{A}$ with \|\|$_{I} \leq\|\|$. If $I \cap \mathcal{A}=(0)$ then \|\|$_{I}$ is a norm and thus \|\|$_{I}=\|\|$. It follows from Lemma 2 that $I=(0)$

(G) This follows from $(F)$.

Pro of of Corollary 1. Every Q-norm on $\mathcal{A}$ dominates a continuous $\mathrm{Q}$-norm on $\mathcal{A}$. It will therefore suffice to show that every continuous $\mathrm{Q}$-norm on $\mathcal{A}$ dominates the complete norm || on $\mathcal{A}$. Assume now that \|\| is a Q-norm on $\mathcal{A}$ and that $\|a\| \leq D|a|$ for all $a \in \mathcal{A}$.

Let $a \in \mathcal{A},|a|=1$. Choose $b \in \mathcal{A}$ such that $|b|=1$ and $\varrho(a b) \geq \varepsilon$ where the constant $\varepsilon>0$ does not depend on $a$. Then

$$
\varepsilon \leq\|a b\| \leq\|a\|\|b\| \leq\|a\| D|b|=D\|a\| .
$$

Thus $|a|=1$ implies $\|a\| \geq \varepsilon / D$. It follows that \|\| dominates the complete norm $\mid 1$.

Proof of Corollary 2. We show $(A) \Rightarrow(D) \Rightarrow(C) \Rightarrow(B) \Rightarrow(A)$ and $(C) \Leftrightarrow(E)$. In the first circle of implications only the following is nontrivial:

$(\mathrm{A}) \Rightarrow(\mathrm{D})$ : Let || be a norm on $\mathcal{A}$ and \|\| the complete norm. By [12, Lemma 3.2] both norms dominate a common norm ||$_{1}$. By Proposition 1, \|\| majorizes a norm ||$_{2}$ which is equivalënt to ||$_{1}$. By minimality, \|\|$=||_{2}$. Hence the complete norm is equivalent to ||$_{1}$ and so || dominates the complete norm.

$(C) \Rightarrow(E)$ : If all norms on $\mathcal{A}$ are $Q$-norms, then $\varrho_{\mathrm{p}}(a)=Q_{\mathcal{A}}(a)$ for all elements $a \in \mathcal{A}$. By semisimplicity of $\mathcal{A}$ every nonzero ideal in $\mathcal{A}$ contains an element $a$ with $\varrho_{\mathcal{A}}(a)>0$.

$(E) \Rightarrow(C)$ : From Theorem $2(C),(E)$ and Proposition 1 it follows as above that every norm on $\mathcal{A}$ dominates the complete norm and is therefore a Q-norm.

\section{References}

[1] 13. Aupetit, The uniqueness of the complete norm topology in Banach algebras and Banach Jortan algebras, J. Finct. Anal. 47 (1982), 1-6.

2] F. IF. Bonsull, A mintimul property of the norm in some Banach algebras, J. London Matili, Soce. 29)(1954), 156164 .

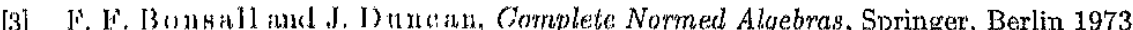

4] S. B. (ILevoland, Homomorphisms of nonn-commutative *-olgebras, Pacific J. Math.

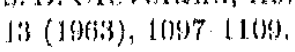

[5] J. Tistorle, Normes d'algrbress minimales, topologie d'algèbre normée minimum sur

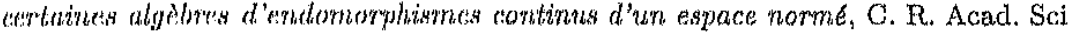
Paria Str. A 277 (1073), 425427.

[6] M. I. May er, Minimnal norms on SQDZ-Banach algebras, Proc. Roy. Irish Acad. 89) A (1980), 127 1333.

(7] , The spetctral externstim property and extension of multiplicative linear function als, Proce. Anwer. Math. Soc. 112 (1991), 855861.

[8] 'T', W. 1'alnuer, Sppectral alyebras, subalgebros and pseudonorms, Rocky Mountain J. Math., to appesar.

[9] T. J. Reatisford, A short proof of Johnson's theorem, Bull. London Math. Soc. 21 $(1989), 487.288$

[10] (C. E. Rickarti, General Theory of Banach Algebras, Krieger, New York 1960.

[11] A. Rodriguca-Palncios, Automatic continuity with application to $C^{*}$-algebras, Math. Proce, Catnloriklge Philos. Scse, to appear.

[12] B. X. To mi In k and I3. Yood, Incomplete romed algebra norms on Banach algebras, Studia Matili. $95(1989), 119132$.

[13] B. Yoocl, Komomorphisms on normed algebrus, Pacific J. Matil. 8 (1.958), 373-381

DHPARTMENT OF MATHEMATTOS ANI COMPUTER SOTENCE

GEORGIA STATE UNIVERSTTY

ATLANTA, GHORGIA 30303

U.S.A.

Rereived May 14, 1991 